\title{
Missbrauchsabbildungen und die Orientierung am Kindeswohl: Finn \& Lina
}

„Das Jugendamt äußerte Bedenken bezüglich der gefundenen Missbrauchsabbildungen. "(Falldokumentation 10.1).

- Zusammenfassung Der Schwerpunkt dieses Kapitels liegt auf der institutionellen Kinder- und Jugendhilfe sowie an diese gekoppelte Fach- und Rechtsbereiche. Im Fokus der Reflexionen stehen Möglichkeiten und Grenzen des Jugendamts, mittels Gefährdungseinschätzung einen Schutz des Kindeswohls vor potenziellen Missbrauchsabbildungen und deren Verbreitung herzustellen. Anschließend werden Herausforderungen der Sachverständigenbegutachtung in familiengerichtlichen Verfahren bei mediatisierter sexualisierter Gewalt behandelt. Das Kapitel ermöglicht angrenzenden Berufsfeldern, Ansätze der Kinder- und Jugendhilfe sowie familiengerichtliche Interventionen nachzuvollziehen.

\section{Fallvignette Finn und Lina - Teil 1}

\section{Gesprächsnotiz, Allgemeiner Sozialer Dienst 02.01.}

Die telefonische Kontaktaufnahme erfolgte am 02. Januar durch Herrn G., Kindesvater (KV) von Finn (10 Jahre) und Lina (3 Jahre). Herr G. lebt getrennt von der Kindesmutter (KM). Herr G. berichtet, dass sein Sohn Finn bei der KM Bilder seiner 3-jährigen Schwester gesehen habe, auf denen diese nackt posiere und spiele. Der Junge habe dies dem KV erzählt 
und sich in dem Gespräch daran erinnert, dass der jetzige Lebensgefährte der KM, Arthur M., vor etwa zwei Jahren auch von Finn Nacktbilder gemacht habe.

Finn sei sehr wütend geworden, so der KV. Der Junge wolle nicht, dass seine Schwester nackt gesehen werden könne. Finn habe sich damals sehr geschämt, als die Fotos von ihm gemacht wurden. Finn wolle die KM und ihren Partner nicht mehr besuchen. Seine Schwester wolle er aber sehen. Herr G. wisse nicht, wie er sich verhalten solle.

\section{Reflexionsfragen}

- Was löst die Notiz in uns aus?

- Welche Assoziationen haben wir zur Situation der Kinder und der Familie?

- Wie kann die Situation fachlich eingeordnet werden?

\subsection{Reflexionen zur fachlichen Einordnung des Fallbeispiels (Teil 1)}

In der Gesprächsnotiz deutet sich ein mediatisierter sexualisierter Übergriff gegen den 10-jährigen Finn und mutmaßlich seine Schwester Lina an. Gemäß den Darstellungen des Kindesvaters wurden Nacktaufnahmen gegen Finns Willen gemacht und sind für den Jungen schambehaftet. Der Lebenspartner der Mutter hätte sich somit in mehrfacher Weise über Finns Widerstand hinweggesetzt: Erstens durch den Eingriff in eine als intim erlebte Situation, zweitens durch das fotografische Festhalten der Situation. Dabei kann der Partner der Kindesmutter Finns Einvernehmen nicht voraussetzen. Der Junge bestimmt nicht mehr über einen Intimbereich und wird entmachtet. Die Missachtung seines fehlenden Einvernehmens sowie die Überwindung der Widerstände Finns definieren die Handlung als Übergriff (Hipp et al., 2017). Unklar bleibt, ob sich in der Bemerkung, ,der Junge wolle nicht, dass seine Schwester nackt gesehen werden könne“, ein mögliches Verbreitungsrisiko, ein Bewusstsein des Jungen für ein bestehendes Verbreitungsrisiko oder Erfahrungen mit der Verbreitung von Nacktaufnahmen andeuten.

Auf eine Integritätsverletzung Linas weist ferner hin, dass im Zusammenhang ihrer Nacktaufnahmen von „Posieren“, dem Einnehmen einer ungewöhnlichen Körperhaltung, gesprochen wird. Sofern die Begrifflichkeit nicht zufällig gewählt 
ist, bleibt ungeklärt, weshalb eine 3-Jährige nackt posiert und hiervon Fotoaufnahmen gemacht werden. Selbst wenn die Pose nicht auf Aufforderung des*der Fotograf*in geschähe, deutete sich in der Fotoaufnahme zumindest ein mangelndes Problembewusstsein an. In den Focus Group Interviews wird deshalb die Möglichkeit gezielter sexualisierter Übergriffe durch den Lebensgefährten der Kindesmutter thematisiert:

„Wobei, was natürlich schon sicherlich auf jeden Fall so ein Verdacht ist, der im Raum steht, dass eben Arthur hier durchaus die Fotos mit sexuellem Interesse macht und wie das zu dem Posieren kommt, dass das wirklich auch auf Aufforderung hin passiert, weil man merkt ja zumindest auch aus dem eigenen Erleben von Finn, dass er das als durchaus grenzüberschreitend erlebt hat.“

Rechtswissenschaftlerin, FGI

\subsubsection{Assoziationen zum Problembewusstsein der Kindeseltern}

Diffus ist, welche Bedeutung der Kindesmutter im Zusammenhang mit den Fotoaufnahmen zukommt. Lediglich die Angabe, Finn wolle die Kindesmutter und den Partner nicht mehr sehen, weist darauf hin, dass der Junge sich dort nicht mehr wohl oder gegen Grenzverletzungen geschützt zu fühlen scheint. In Focus Group Interviews werden zudem Hypothesen zum Verhältnis der Kindeseltern untereinander sowie zur Bedeutung des Verhältnisses für die fachliche Einschätzung der Darstellungen Herrn G.s diskutiert:

\footnotetext{
„Also wenn wir hochstrittige Eltern hätten, dann würde der Vater anders agieren. Der würde sofort seine Frau beschuldigen, der würde sofort sagen, ,Ich will nicht mehr, dass die Kinder da sind, die Mutter ist nicht in der Lage, die Kinder zu schützen' [...]. Und da agiert er ja eher so ein bisschen ruhiger auch.“

Kinder- und Jugendpsychotherapeutin, spezialisierte Fachberatung, FGI

„Und gleichzeitig, wenn der [Kindesvater] hoch emotional wäre, könnte man das in der Situation auch absolut nachvollziehen."

Rechtswissenschaftlerin, FGI
}

Die Expert*innen schlussfolgern, dass ein konfliktbehaftetes Verhältnis der Kindeseltern zwar in Erwägung gezogen werden müsse. Es bestehe zugleich das Risiko, dem Kindesvater irrtümlicherweise zu unterstellen, er instrumentalisiere 
das Thema „sexueller Missbrauch“ etwa im Rahmen eines Umgangs- oder Sorgerechtsstreits. Trotz beim Vater wahrgenommener Unklarheit und Unsicherheiten wird der Vater von den Expert*innen vorerst als kooperationsfähig eingeschätzt. Eine potenzielle Gefährdung durch die Kindesmutter könne dagegen weniger gut eingeschätzt werden.

„Die Rolle der Mutter ist hier ja sehr unklar. Also bei der Mutter kann es ja alles sein.
Jetzt irgendwie zu sagen, man macht jetzt gleich eine Sorgerechtsregelung, erschiene
mir ein bisschen zu schnell, weil/ also wir haben jetzt Fantasien, was der Arthur
gemacht hat und wie die Mutter daran beteiligt ist, könnte aber auch einfach sein
quasi, dass es der an Problembewusstsein fehlt oder aber auch an Stärke quasi. [...]
Man muss erst mal einschätzen, wie schwerwiegend ist der Vorwurf.“

Rechtswissenschaftlerin, FGI

Nichtsdestotrotz handelt es sich bei der Gesprächsnotiz des Jugendamts um die Protokollierung eines Anrufs durch den Kindesvater und nicht um eine Rekonstruktion der kindlichen Sichtweisen. Der Fokus der Notiz liegt also nur indirekt auf dem Erleben und den Bedürfnissen des primären Adressaten. Ausgehend davon, dass der Kindesvater die Perspektive Finns sinngemäß wiedergibt, wird Finn in den Focus Group Interviews als bedürfnisorientiert wahrgenommen. Sein Unrechtsbewusstsein und die Fähigkeit, seine Gefühle auszudrücken, bewerten die Expert*innen als Hinweis darauf, dass man mit dem Jungen arbeiten könne.

\subsubsection{Assoziationen zum Gewaltausmaß}

Dabei kann der Anruf beim Jugendamt (JA) sowohl auf sexualisierte Übergriffe wie auf einen sexuellen Kindesmissbrauch im Sinne § 176 StGB hinweisen, dessen Schwere jenseits des Erlebens der Kinder durch die Möglichkeit von Missbrauchsabbildungen sowie deren Verbreitung bestimmt wird. Für diesen Fall ist von einem gesteigerten Gefährdungsrisiko des Kindeswohls auszugehen.

„Dieses Verbreitungsrisiko [...], wenn die Bilder einmal online waren, ist die Frage, ob er es selbst verbreitet oder nicht, wahrscheinlich für die Einschätzung der Gefährdung der Kinder gar nicht mal so relevant. Sondern der Akt als solcher, dass er Bilder ins Netz stellen könnte, würde ja schon ausreichen.“

Rechtswissenschaftlerin, FGI 
Auch ohne sexuelle Handlungen am Kind wäre ein Verleiten zu bestimmten sexualisierten Körperstellungen als sexueller Missbrauch strafbar. In diesem Fall würden die Fotos auch den Straftatbestand kinderpornografischer Inhalte ${ }^{1}$ erfüllen. Sodann spielt noch eine Rolle, ob die Bilder verbreitet wurden bzw. werden sollen.

„Es geht auch ein Stück weit um eine Strafverschärfung. Der Besitz [Anm.: kinderpornografischer Inhalte] ist ja jetzt nicht so hoch sanktioniert wie die Verbreitung von entsprechendem Material. Und auch das muss eben bewiesen werden und belegt werden."

Spezialermittler Cybercrime, FGI

Eine strafrechtliche Bewertung kann im vorliegenden Fall relevant werden. Sie ist aber nicht identisch mit der Orientierung am Kindeswohl. Zwar sollte das Kindeswohl bei ermittlungstaktischen und strafrechtlichen Erwägungen dringend berücksichtigt werden. Nichtsdestotrotz folgen Kindeswohlorientierung und Strafverfolgung unterschiedlichen Auftragslagen (Gerber, 2006). Umgekehrt sind die Möglichkeiten der Kinder- und Jugendhilfe sowie der zivilrechtlichen Eindämmung von Missbrauchsabbildungen begrenzt. Dies führt bisweilen zu Abwägungsprozessen. Da der Anruf beim Jugendamt eingeht, orientieren wir uns weiterhin an der Handlungslogik des Jugendamts.

\subsection{Handlungsempfehlungen (Teil 1)}

\subsubsection{Die Gefährdungseinschätzung orientiert sich am Kindeswohl}

Nach dem Anruf Herrn G.s liegen die Fallverantwortung und der Handlungsauftrag beim Jugendamt. Das Jugendamt ist als öffentlicher Träger der Kinder- und

${ }^{1} \S 184$ b Abs. 1, 2. HS StGB definiert einen kinderpornografischen Inhalt als Darstellung, die a) sexuelle Handlungen an, vor oder mit einem Kind, b) ein ganz oder teils unbekleidetes Kind in geschlechtsbezogener Haltung oder c) Genitalien bzw. das Gesäß von Kindern in sexuell aufreizender Form wiedergibt respektive zeigt. 
Jugendhilfe dem Kindeswohl verpflichtet ${ }^{2}$. Der Anruf liefert gewichtige Anhaltspunkte für eine Kindeswohlgefährdung Finns und Linas aufgrund des Verdachts auf sexuellen Missbrauch durch den Partner der Kindesmutter und/oder die Kindesmutter. Dem Jugendamt kommt nach § 8a SGB VIII ein Schutzauftrag bei Kindeswohlgefährdung zu. Das Amt ist gefordert, ein Gefährdungsrisiko unter Einbezug mehrerer Fachkräfte einzuschätzen, wenn ihm gewichtige Anhaltspunkte für die Gefährdung des Wohls eines Kindes bekannt werden. Diese können unterschieden werden nach Formen der Schädigung, Ursachenkonstellationen (z. B. massiven Elternkonflikten) oder Gefährdungskontexten (z. B. restriktive weltanschauliche Gemeinschaften (Schone, 2017). Im vorliegenden Fall liegt eine Kindeswohlgefährdung vor, wenn die Kindesmutter a) über kein ausreichendes Problembewusstsein zu möglichen mediatisierten sexualisierten Übergriffen verfügt, b) nicht in der Lage ist, Übergriffe gegen Finn und Lina abzuwenden oder c) selbst sexualisiert übergriffig handelt. Damit wäre vorerst auch ihre Kooperationsfähigkeit fraglich. Potenziell betroffen sind mehrere (zwei) Kinder, eines davon im Vorschulalter. Bei Gefährdungseinschätzungen wird gemeinhin davon ausgegangen, dass ein Risiko als umso größer einzuschätzen ist, je mehr Kinder betroffen und je jünger betroffene Kinder sind. Junge Kinder gelten aufgrund ihrer Schutzlosigkeit und ihres psychischen und körperlichen Entwicklungsstandes als besonders vulnerabel, da sich Schädigungen besonders massiv und biografisch folgenreich auswirken können (Bathke et al., 2019; Lillig, 2006). Zudem ist ungeklärt, ob Nacktaufnahmen Finns und Linas verbreitet wurden. Das Belastungsrisiko für Betroffene ist im Falle einer Verbreitung von sexualisierten Foto- und Videoaufnahmen oder gespeicherten sexualisierten Kommunikationsverläufen aufgrund der Möglichkeit, davon wiedereingeholt zu werden, als erhöht zu bewerten (Kärgel \& Vobbe, 2020). Siehe zur Problematik von Verbreitung und Wiedereinholung Kap. 8.

\footnotetext{
${ }^{2}$ Das Kindeswohl ist ein unbestimmter Begriff des Familienrechts. Zweck der Unbestimmtheit des Begriffs ist die Möglichkeit seiner kontextabhängigen Auslegung und Weiterentwicklung. Ein Mindestkonsens besteht darin, dass im „Wohl des Kindes“ sei, was für das Kind am besten ist (Bathke et al., 2019). § 1 Abs. 1 SGB VIII formuliert daher als Ziel der Kinder- und Jugendhilfe, dass jeder junge Mensch das Recht auf Förderung seiner Entwicklung und auf Erziehung zu einer eigenverantwortlichen und gemeinschaftsfähigen Persönlichkeit hat.
} 


\subsubsection{Beteiligung der Kinder und Erziehungsberechtigten}

Bei der Gefährdungseinschätzung sind laut § 8a Abs. 1 SGB VIII sowohl die Kinder selbst als auch die Erziehungsberechtigten miteinzubeziehen, sofern dadurch der wirksame Schutz des Kindes/der Kinder nicht infrage steht. Zweifelhaft ist, ob ein unmittelbarer Einbezug der Kindesmutter den wirksamen Schutz der Kinder infrage stellen könnte, da sie als Mittäterin Schweigegebote oder Druck auf die Kinder verstärken könnte. In den Focus Group Interviews entsteht hieraus ein Dissens.

„Ich finde auch wichtig, die Mutter ins Boot zu nehmen, auch um zu sehen, was für eine Haltung hat sie zu Arthur. Kann ja sein, sie hat eh einen Verdacht, sie findet den sowieso komisch und sie entscheidet sich, sich von ihm zu trennen."

Kinder- und Jugendpsychotherapeutin, spezialisierte Fachberatung, FGI

„Im Zweifelsfall quasi kann man ja vom gemeinschaftlichen Sorgerecht ausgehen, dass unter Umständen es hier ausreichen würde, jetzt den Vater, der sich sowieso jetzt an das Jugendamt wendet, miteinzubeziehen in die Gefährdungseinschätzung und vielleicht aber mit der Mutter erst mal nicht das Gespräch zu suchen, weil da eben das Risiko relativ hoch ist, dass sie sich mit dem Partner solidarisiert. [...] Wie kann man jetzt vorgehen? Man spricht mit Finn, wen bindet man von den Familienangehörigen noch weiter ein?“

Rechtswissenschaftlerin, FGI

Da ein potenzielles Risiko durch die Mutter erst mit weiteren Eindrücken von der Familien- und Erziehungssituation einzuschätzen ist, sollte sie nicht unmittelbar, aber so bald wie möglich einbezogen werden. Ein Einbezug des Jungen erscheint demgegenüber prioritär und löst womöglich die Frage, wann die Kindesmutter einbezogen werden sollte. Ein Hinzuziehen spezialisierter Fachkräfte erscheint im Zuge der Beteiligung Finns sinnvoll, weil diese qua Funktion und Ausbildung über die notwendige Sensibilität im Umgang mit Betroffenen sowie über Kompetenzen der adäquaten Durchführung von Erstgesprächen zur Gefährdungseinschätzung verfügen. Jugendämter sind prinzipiell aufgefordert, die Gefährdungseinschätzung im Zusammenwirken mehrerer Fachkräfte vorzunehmen und Zuständigkeiten durch aufbau- und ablauforganisatorische Maßnahmen festzulegen (Bathke et al., 2019). Dazu sind Fachkräfte oder Organisationseinheiten zu benennen, die standardmäßig an der Gefährdungseinschätzung beteiligt werden. Prinzipiell kann dies auch unter Delegation von Teilaufgaben an externe Fachberatungsstellen, die zu sexualisierter Gewalt arbeiten, geschehen (Igney \& Monz, 2020). 
Im Kontakt mit Finn kann abgeklärt werden, wie dieser die Situation erlebt, was seine Bedürfnisse sind, inwieweit seine Bedürfnisse in der Familie berücksichtigt und wann diese oder seine Persönlichkeitsrechte missachtet werden. Daraus werden idealerweise Eindrücke von der Erziehungsfähigkeit der Eltern, vor allem der Mutter, von der Haltung der Mutter zu mediatisierten Übergriffen, ihrer Einstellung gegenüber dem übergriffigen Verhalten des Lebenspartners sowie Möglichkeiten, die Kindesmutter in den Schutz der Kinder einzubeziehen, abgeleitet. Zudem sollte mit Finn hinsichtlich der Frage gearbeitet werden, welche Wünsche er bezüglich einer Veränderung der Situation hat. Nicht minder wichtig ist es in der Beratung Finns und der Kindeseltern, das sexualisiert übergriffige Verhalten Arthurs sowie das Verhalten der Kindesmutter einzuschätzen. Dabei ist zu beachten, dass das Thema für Finn schambesetzt und nicht gleichbedeutend mit seinen Gesprächswünschen ist.

Die Gefährdungseinschätzung bezieht sich nicht ausschließlich auf Finn, sondern auch auf Lina, die offenbar bei der Kindesmutter lebt. Sie ist eine 3-Jährige, wodurch ein gesprächsbasierter Einbezug des Mädchens ohne besondere Ausbildung erschwert wird. In den Focus Group Interviews wird daher die Möglichkeit in Erwägung gezogen, die Kindertagesstätte, die Lina besucht, einzubeziehen.

\subsubsection{Weitere Handlungsschritte in Wenn-Dann-Zusammenhängen}

Die Zahl der Unbekannten beim aktuellen Kenntnisstand führt dazu, dass weitere Handlungsschritte als Wenn-Dann-Zusammenhänge dargestellt werden. Gemeint ist, dass Handlungsansätze für ein verschiedentliches Ausfallen der Gefährdungseinschätzung skizziert werden (s. Abb. 10.1). Die Varianten werden ausschließlich angerissen, weil das Fallbeispiel im Folgeverlauf noch erweitert wird.

\subsubsection{Das Jugendamt kann keine Gefährdung feststellen}

Das Jugendamt kann die Situation nicht als akute Gefährdung bewerten, beispielsweise weil Finn und gegebenenfalls auch Lina die Darstellungen des Vaters relativieren und weitere Hinweise auf Belastungen, Vernachlässigung oder Missbrauch fehlen. Oder: Die Kindesmutter bestätigt im Zusammenhang der gemeinsamen Erörterungen eines Gefährdungsrisikos zwar, dass Fotoaufnahmen der Kinder gemacht wurden, auf denen diese nackt zu sehen sind. Sie zeigt sich aber einsichtig und signalisiert, sie könne nachvollziehen, dass es sich hierbei um Grenzverletzungen handelt. Jedoch sei der Kontext nicht sexualisiert übergriffig konnotiert gewesen. Die sich so darstellende Situation ist für das Jugendamt 


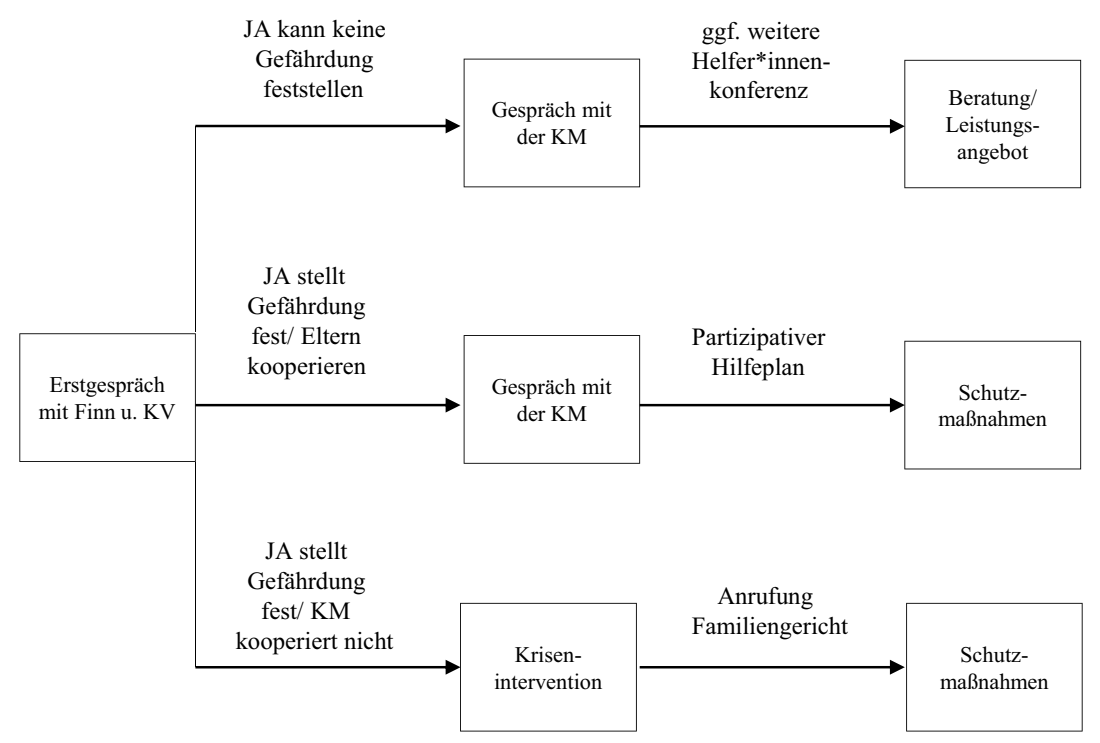

Abb. 10.1 Handlungsschritte

denkbar schwierig. Eine Gefährdung ist nicht eindeutig ausgeräumt, schließlich ist vorstellbar, dass Finn und seine Schwester von Schweigegeboten geleitet werden bzw. die Mutter ein gewalttätiges Verhalten bagatellisiert. Eine Befreiung von der Handlungsnotwendigkeit bedeutet dies für den Allgemeinen Sozialen Dienst (ASD) also nicht, wenngleich die Unterstützung der Kinder stärker von der Kooperationsbereitschaft der Familie abhängt. Denkbare Schritte sind unter dieser Prämisse:

- weitere Helfer*innenkonferenzen unter Einbeziehung von Schule und Kindertagesstätte,

- Leistungsangebote der Kinder- und Jugendhilfe - beispielsweise ambulante Hilfen zur Erziehung - mit besonderem Fokus auf Finn und Lina,

- optional der Hinweis, dass bereits die Fotos gegen den Willen der Kinder eine Straftat nach § 201a StGB (,Verletzung des höchstpersönlichen Lebensbereichs durch Bildaufnahmen") darstellen und gegebenenfalls eine Anzeige gegen den Partner der Kindesmutter erstattet werden muss, sollten solche erneut angefertigt oder gar verbreitet werden, 
- eine Beurteilung des Problembewusstseins und der Kooperations- und Erziehungsfähigkeit der Eltern anhand der Frage, ob sie ihre Kinder bei der Inanspruchnahme von Hilfen zur Erziehung oder der Unterlassungsaufforderung angemessen vertreten,

- Finn und Lina sind im Sinne von Empowerment bei der Wahrnehmung ihrer Rechte zu bestärken und für den Fall eventuell folgender oder massiverer (mediatisierter) sexualisierter Gewalthandlungen Anknüpfungspunkte einer Unterstützung proaktiv aufzuzeigen.

\subsubsection{Das Jugendamt stellt eine Gefährdung fest, die Eltern wollen den Schutz der Kinder herstellen}

Das Jugendamt stellt ein Gefährdungsrisiko durch den Partner der Kindesmutter fest, das idealerweise von den Eltern, insbesondere der Kindesmutter erkannt wird. Sodann besteht die Möglichkeit, die Kindeseltern beim effektiven Schutz von Finn und Lina zu unterstützen und auf die Inanspruchnahme von Hilfen hinzuwirken. $\mathrm{Zu}$ diesem Zweck wird den Eltern Beratung angeboten. Aufgrund der Gewalt gegen die eigenen Kinder durch eine Vertrauensperson müssen die Kindeseltern selbst als Betroffene behandelt werden (Bange, 2011). Das Jugendamt kann im Rahmen seines Leistungsangebots Aufgaben delegieren, auch hier beispielsweise an eine Fachberatungsstelle, die auf sexualisierte Gewalt spezialisiert ist (Igney \& Monz, 2020).

In der Verantwortung des Jugendamts liegt die gemeinschaftliche Entwicklung eines Hilfeplans mit den Eltern ( $\$ 36$ SGB VIII). Hierbei ist der sozialräumliche Schutz Finns und Linas sicherzustellen. Sozialräumlich heißt in diesem Fall sowohl örtlich als auch digital, weil von einer mediatisierten Integritätsverletzung auszugehen ist bzw. Arthur M. sich weiterhin mittels Kommunikationsmedien übergriffig verhalten könnte. Die Zuständigkeit in derartigen Gewaltschutzsachen liegt beim Familiengericht. Dort kann die Kindesmutter vor dem Hintergrund der Kindeswohlgefährdung durch den Kindespartner auf Basis von § 1666 BGB einen (Eil-)Antrag auf gerichtliche Schutzmaßnahmen stellen, welcher umfasst, dass der Partner der Kindesmutter im Falle einer gemeinsamen Wohnung, in der auch die Kinder leben, diese verlassen muss ( 2 Abs. 1 Satz 1 GewSchG) und er keinen Kontakt zu den Kindern aufnehmen darf - auch nicht unter Verwendung von „Fernkommunikationsmitteln“ (§ 1 Abs. 1 Satz 4). Der entsprechende Antrag müsste durch die Eltern stellvertretend für die Kinder gestellt und könnte gegen Arthur M. per Eilverfahren durchgesetzt werden. Verfahren, die eine Gefährdung 
des Kindeswohls betreffen, sind nach $\S 50$ e Abs. 1 FGG beschleunigt durchzuführen. ${ }^{3}$ Das Vorgehen ermöglichte es, Finn und Lina auch ohne Strafprozess vor unmittelbarer Gewalteinwirkung zu schützen. Über eine Strafanzeige kann im Rahmen fortlaufender Beratung und abhängig von den zu erwartenden Belastungen für die Kinder sowie von der Aussicht auf einen Mehrwert des Verfahrens entschieden werden. Freilich ist damit zu rechnen, dass Arthur M. zwischenzeitlich mögliche Indizien einer mediatisierten sexualisierten Gewalt vernichtet, schließlich würde er durch den Antrag beim Familiengericht gewarnt. Desgleichen wäre eine Weiterverbreitung möglicher (Missbrauchs-)Abbildungen Finns und Linas durch die Gewaltschutzanordnung oder, soweit der Ort einer Veröffentlichung bekannt ist, durch eine Beschwerde bei jugendschutz.net (s. Kap. 8) zu begrenzen.

\subsubsection{Das Jugendamt stellt eine Gefährdung fest, die Kindesmutter beteiligt sich nicht am Schutz der Kinder}

Das Jugendamt stellt eine Gefährdung durch den Partner der Kindesmutter fest, die von der Kindesmutter nicht erkannt oder bagatellisiert wird oder an der die Kindesmutter selbst beteiligt ist. Interventionen zugunsten von Kindern nach dem GewSchG sind bei Gefährdung durch Elternteile ausgeschlossen. § 8a SGB VIII verlangt das Angebot von Hilfen an die Erziehungsberechtigten und damit die Erarbeitung eines Schutzkonzeptes. Wenn dies mangels Kooperation nicht möglich ist, ist das Familiengericht anzurufen. Mit § 1666 BGB kommt dem Familiengericht die Aufgabe zu, über Maßnahmen zur Abwendung einer Kindeswohlgefährdung zu entscheiden, wenn Eltern, hier die Mutter, nicht gewillt oder in der Lage sind, die Gefahr abzuwenden. Das Familiengericht hat nach $\S 1666$ Abs. 4. in Angelegenheiten der Personensorge auch die Möglichkeit, Maßnahmen gegen Dritte, hier den Partner der Kindesmutter, zu verhängen. Auch dazu können Verbote gehören, Kontakt zum Kind aufzunehmen, Zusammentreffen mit dem Kind herbeizuführen, auf unbestimmte Zeit eine Wohnung zu nutzen oder bestimmte Orte aufzusuchen, an denen sich das Kind aufhält. In allen Fällen sind digitale Medien ausdrücklich als kindlicher Sozialraum zu bedenken.

Der Kindesmutter kann zugleich die Inanspruchnahme von Leistungen der Kinder- und Jugendhilfe zur Auflage gemacht werden, beispielsweise durch eine spezialisierte Fachberatungsstelle gegen sexualisierte Gewalt in Kindheit und Jugend. Ziel einer derartigen Intervention ist die Sensibilisierung der Mutter für

\footnotetext{
${ }^{3}$ Fachberater*innen berichteten im HUMAN-Projekt, dass sie in der Praxis sehr unterschiedliche Erfahrungen machen. Dazu gehört auch, dass beschleunigte Verfahren oftmals nicht in der vorgesehenen Zeit durchgeführt werden.
} 
die aus den sexualisierten Aufnahmen folgenden Belastungen sowie die Möglichkeit weiterer schwerwiegender sexualisierter Übergriffe gegen die Kinder. Fraglich ist, inwieweit der Kontakt der Kindesmutter mit den Kindern bis auf weiteres beschränkt werden muss, wenn die Mutter aufgrund fehlender Gewalteinsicht nicht dafür Sorge trägt, dass ihr Partner die Kontaktverbote zu Finn und Lina einhält. Denkbar wäre eine Übertragung des Aufenthaltsbestimmungsrechts auf den $\mathrm{KV}$, ein (vorübergehender) Wechsel in den väterlichen Haushalt und die Anordnung vorerst befristet begleiteter Umgangskontakte mit der Kindesmutter auf Basis von $\S 1684$ Abs. 4 Satz 3 und 4. Weitere Regelungen könnten anschließend davon abhängig gemacht werden, wie die Kindesmutter ihre elterliche Sorge im Rahmen der Kontakte wahrnimmt, wie sich ihre Problemeinsicht entwickelt und wie sie den Schutz der Kinder vor weiteren Übergriffen des Partners sicherstellt.

\subsubsection{Möglichkeiten der Strafverfolgung und Strafgerichtsbarkeit}

Die mediatisierten Aspekte der Gewalt - etwaige Missbrauchsabbildungen sowie ein Nachvollzug deren möglicher Verbreitung - können nur durch Strafverfolgungsbehörden ermittelt werden.

„Ein Anfangsverdacht dürfte gegeben sein, da kriegt man relativ schnell dann entsprechende Beschlüsse, zu gucken, welche Seiten hat er besucht auf dem Rechner, welche Internetseiten. [...] Also den Verlauf sich anzugucken, die Speicher sich anzugucken.“

Spezialermittler Cybercrime, FGI

„Wir nehmen ein Video und errechnen uns da den Fingerabdruck, den Hashwert, und wir suchen mittels dem Hashwert nach dieser Datei.“

Spezialermittler Cybercrime, FGI

Mitarbeitende des Jugendamtes sind durch § 203 Abs. 1 StGB zunächst als Geheimnisträger*innen zu verstehen. Die Möglichkeit einer Strafanzeige durch das Jugendamt ergibt sich jedoch entweder durch die Einwilligung der Beratenen - in gesetzlicher Stellvertretung der Kinder, auch der Kindeseltern - oder durch einen sogenannten rechtfertigenden Notstand, wie ihn $\S 34$ StGB definiert. Dabei sind die Voraussetzungen vergleichbar mit $\S 8$ a Abs. 3 SGB VIII (Kliemann \& Fegert, 2015). Ein rechtfertigender Notstand könnte gegebenenfalls damit begründet werden, dass das Jugendamt und das Familiengericht einer Verbreitung von Missbrauchsabbildungen durch den Partner in Täter*innen-Netzwerken technisch nicht in derselben Weise entgegenwirken können wie spezialisierte 
Strafermittler*innen mittels o. g. Hashwertsuche. Mit der Verbreitung der Aufnahmen erhöht sich das Risiko einer Reviktimisierung der Kinder (Konsum der Missbrauchsabbildungen durch Dritte) und multipler Folgebelastungen wie Ängste vor erneuter Konfrontation mit den Gewaltabbildungen (s. Kap. 8 und 9). Weil jedoch auch die Polizei kaum mit Gewissheit alle Datenträger beschlagnahmen kann, möchten wir das Einvernehmen der Gewaltbetroffenen, insbesondere Finns, seine möglichen Belastungen und die Aussicht auf den Erfolg einer Strafanzeige im Abwägungsprozess unbedingt zu berücksichtigen geben. Dasselbe gilt, wenn die Kindeseltern, besonders der Kindesvater, zum Stellen einer Strafanzeige beraten werden. Idealerweise wird diese Beratung durch eine*n Nebenklagevertreter*in unterstützt.

Im Rahmen des Strafverfahrens wird eruiert, inwieweit Handlungen der Kindesmutter oder des Partners der Kindesmutter den Straftatbestand des sexuellen Kindesmissbrauchs ( $§ 176$ StGB) - aufgrund gemeinschaftlicher Handlungen oder der Herstellung von Missbrauchsabbildungen - schweren sexuellen Kindesmissbrauchs ( $\$ 176 \mathrm{a}$ StGB) und/oder der Verbreitung kinderpornografischer Inhalte (§ 184b StGB) erfüllen.

Im Kontext der Strafverfolgung und Strafgerichtsbarkeit ist das Kindeswohl zu gewährleisten. Dies geschieht idealerweise, indem Kinder durch geschultes Personal altersgerecht vernommen werden. Wenn während der Befragung darüber hinaus ein Audio- und Videomitschnitt erfolgt, können gegebenenfalls Mehrfachbefragungen betroffener Kinder und Jugendlicher vermieden werden ${ }^{4}$ (Bundeskoordinierung Spezialisierter Fachberatung gegen sexualisierte Gewalt in Kindheit und Jugend, 2020). Dabei ist zu berücksichtigen, dass Video- und Audioaufzeichnungen triggern und Belastungen reaktivieren können. Kinder sind deswegen über den Sinn der Aufnahme und ihre Rechte zu informieren. Sofern ihnen dabei deutlich gemacht wird, dass die Aufzeichnung dem Zwecke der Strafverfolgung dient, kann der Video-Ton-Befragung eine präventive Bedeutung zukommen. Trotzdem können die Aufnahmen zum Beispiel auch gegen den Willen der Kinder in der Hauptverhandlung gezeigt oder an die Verteidigung gegeben werden, was als erneutes Belastungspotenzial zu berücksichtigen ist.

Im Zusammenhang von Straftaten nach $\S 176$ StGB haben Betroffene einen Anspruch auf kostenfreie psychosoziale Prozessbegleitung (Bundesministerium der Justiz und für Verbraucherschutz, 2019). Bei Verdacht auf Gewalt durch Elternteile ist eine Ergänzungspflegschaft nach § 1907 BGB zu bestellen (Bley et al., 2020). Parallel sind eventuelle familiengerichtliche Entscheidungen nicht

\footnotetext{
${ }^{4}$ Leider ergibt sich aus der Videovernehmung kein Anspruch auf den Verzicht von Mehrfachvernehmungen. Die Praxis in Deutschland ist diesbezüglich sehr unterschiedlich.
} 
von einer Bestrafung des*der Täter*innen abhängig zu machen. Dies wird im Anschluss an die nun folgende Fortsetzung des Fallbeispiels diskutiert.

\section{Fallvignette Finn und Lina - Teil 2}

\section{Auszug aus dem familienpsychologischen Sachverständigengutachten in der Familiensache \\ 15. September}

iv. Gibt es andere Umstände und Gegebenheiten im Umfeld der Kinder bzw. der Eltern, die jeweils positive oder negative Auswirkungen auf die psychische oder körperliche Entwicklung des Kindes erwarten lassen?

Der im Haushalt der Kindesmutter lebende und für sexuellen Kindesmissbrauch (im Internet) vorbestrafte Arthur M weist nach psychologischer Test- u. Fragebogenuntersuchung $\mathrm{zu}$ Kontrollüberzeugung (Locus of Control), Impulsivität (BIS-II) und Opferempathie (VED) ein geringes Rückfallrisiko auf. Pädophile Veranlagungen (ICD-10, F65.4) manifestieren sich demnach ausschließlich in mittelbaren Formen von Dissexualität, die auf Online-Kontexte beschränkt sind.

Bei den gefundenen Darstellungen von Lina G handelt es sich polizeilichen Einschätzungen zufolge um , auffälliges` Bildmaterial, aber nicht um kinderpornografische Inhalte im strafrechtlichen Sinne. Urheber der Aufnahmen ist unklar. Die Kindesmutter sowie Arthur M Besitz der Aufnahmen. Bildaufnahmen von Finn G $\quad$ sind hinzukommend nicht auffindbar. Von negativen Auswirkungen auf die psychische oder körperliche Entwicklung der Kinder durch Arthur M kann daher nicht zweifelsfrei ausgegangen werden.

\section{Reflexionsfragen}

- Was löst der Gutachtenauszug in uns aus?

- Wie bewerten wir die Professionalität des Gutachtens?

- Wie kann die Situation fachlich eingeordnet werden?

\subsection{Reflexionen zur fachlichen Einordnung des Fallbeispiels (Teil 2)}

Zwischen dem ersten und dem zweiten Fallausschnitt liegen mehrere Monate. Zwischenzeitlich hat offenbar eine Anrufung des Familiengerichts stattgefunden. Außerdem wurde vom Gericht per Beweisbeschluss ein Gutachten in Auftrag 
gegeben. Der Beweisbeschluss ist eine gerichtliche Anordnung im Rahmen der Beweisaufnahme, die dazu dient, Aufgaben zur sogenannten Feststellung von Tatsachen zu delegieren, ,die einer eigenen Tatsachenfeststellung durch das Gericht mangels eigener Sachkunde entzogen sind.“ (Bergmann, 2016, S. 365). Beweisbeschlüsse enthalten in aller Regel einen Kanon sich ergänzender Fragen, die auf möglichst differenzierte und konkrete Tatsachenfeststellung durch eine Begutachtung hinwirken sollen. Im obigen Ausschnitt weist die Nummerierung (iv) darauf hin. Fachhypothesen, die mit dem Fragenkanon hergeleitet werden sollen, müssen vom Gericht als Anknüpfungstatsachen unter einen zu klärenden Gesetzestatbestand subsumiert werden können. Die von dem*der Sachverständigen (fortan auch Gutachter*in) formulierten Hypothesen sollen daher fachwissenschaftlichen Standards folgen und in ihrer Schlussfolgerung nachvollziehbar sein (ebd.).

Durch die im Auszug dargestellte Frageformulierung wird der*die Gutachter*in mitunter ermächtigt, Personen im Umfeld der Kinder und Kindeseltern in die Begutachtung einzubeziehen und fachwissenschaftliche Aussagen über deren Auswirkungen auf die Kindesentwicklung zu treffen. Die Frageformulierung des Beweisbeschlusses (,Gibt es andere Umstände und Gegebenheiten im Umfeld der Kinder bzw. der Eltern ...") ist aus juristischer Perspektive zulässig. Offenbar definiert das Gericht die psychische und körperliche Entwicklung des Kindes als eine Anknüpfungstatsache des unbestimmten Rechtsbegriffs des Kindeswohls. Es gibt dazu auch die Prüfung nicht unmittelbar in den Eltern begründeter Faktoren in Auftrag, da sie im vorliegenden Fall relevant sind. Das Gutachten bezieht folglich Stellung zum im Haushalt der Kindesmutter lebenden Partner. Es impliziert die Einschätzung seines Gefährdungspotenzials unter Rückgriff auf eine sexualmedizinische kriminologische Terminologie (sog. pädophile Veranlagungen, Dissexualität ${ }^{5}$ ) sowie Bewertungen beim Partner und der Kindesmutter (nicht) gefundener Fotoaufnahmen der Kinder. Ein mögliches Rückfallrisiko des wegen sexuellen Kindesmissbrauchs vorbestraften Partners wird im Gutachten unter Verweis auf psychologische Test- und Fragebogenuntersuchung begründet und als gering dargestellt. Bei der Einschätzung pädophiler Veranlagungen verweist das Gutachten auf das Klassifikationssystem der ICD-10.

Die Einschätzung, dass sich pädophile Veranlagungen ausschließlich in mittelbaren Formen von Dissexualität in Online-Kontexten äußern, scheint sich zum

5 „Das Konzept der Dissexualität entstand in Anlehnung an die Definition der Dissozialität als Sozialversagen, da sexuelles Fehlverhalten grundsätzlich zunächst eine gestörte soziale Dimension von Sexualität zum Ausdruck bringt [...]. Dementsprechend kann sexuelles Verhalten dann als dissexuell eingestuft werden, wenn es die Integrität und Individualität eines anderen Individuums verletzt und/oder nicht einwilligende Personen beinhaltet." (Kuhle et al., 2018, S. 19). 
Teil aus der Untersuchung eines Rückfallrisikos zu ergeben, wird sonst aber nicht weiter begründet. Unklar ist, was mit mittelbaren Formen von Dissexualität konkret gemeint ist. Denkbar wäre, dass Arthur M. Missbrauchsabbildungen konsumiert. Dies wird im Gutachten jedoch nicht angeführt. Mehrdeutig bleibt auch die Bezugnahme auf die von Lina gefundenen Aufnahmen. Schon die Formulierung ,auffälliges Bildmaterial“ ist nicht spezifisch. Ferner bleibt diffus, mit welchem Motiv der*die Gutachter*in die Fotoaufnahmen thematisiert. Ein Auftrag zur strafrechtlichen Bewertung von Fotos gehört nicht zur Tatsachenfeststellung familienpsychologischer Gutachten. Zusammenhänge beispielsweise zwischen dem Besitz augenscheinlich grenzverletzender Fotoaufnahmen sowie den Andeutungen einer ,sexuellen Verhaltensstörung' (Dissexualität) Arthur M.s in Online-Kontexten, die den Verweis auf die Art des Materials eventuell legitimieren könnten, werden argumentativ nicht hergestellt. So stehen die Informationen ohne erkennbaren Zusammenhang nebeneinander. In der Zusammenschau suggerieren sie lediglich, dass für Finn und Lina keine akute Gefahr vom Partner der Kindesmutter ausgehe (,geringes Rückfallrisiko“, „Urheber unklar“, ,,auf Online-Kontexte beschränkt“, ,nicht kinderpornografische Inhalte“, „,von negativen Auswirkungen kann nicht zweifelsfrei ausgegangen werden“). Das Gutachten folgt latent dem für Strafverfahren entwickelten Grundsatz in dubio pro reo (im Zweifel für den Angeklagten), als sei eine Gefährdungseinschätzung davon abhängig, dass jeder Zweifel an einer Täterschaft Arthur M.s ausgeräumt werden müsse.

\section{Irrtümliche Bewertung mediatisierter sexualisierter Gewalt im Sachverstän- digengutachten}

Die Sexualmedizin und Sexualforensik beschäftigen sich seit einigen Jahren mit dem Verhältnis von sexualisierter Online-Gewalt zu anderen Formen sexualisierter Gewalt gegen Kinder und Jugendliche. In diesem Zusammenhang werden Unterscheidungen und Überschneidungen des Konsums von Missbrauchsabbildungen und sexuellen Kindesmissbrauchs besonders in den Blick genommen. Verschiedene Studien verweisen darauf, dass manche Menschen Missbrauchsabbildungen konsumieren, um Spannungen abzubauen, die sonst zu Hands-on-Taten ${ }^{6}$ führen könnten (Carter et al., 1987; Riegel, 2004). Neuere Metaanalysen der Dunkelfeldforschung zeigen zugleich, dass der größere Teil (55\%) der Konsument*innen von

\footnotetext{
${ }^{6}$ Hands-on-Gewalt umfasst auch Handlungen, bei denen ein Kind in digitalen Kontexten aufgefordert wird, zu masturbieren oder sich nackt $\mathrm{zu}$ fotografieren. Entscheidend ist der interaktionistische Einbezug des Kindes (Lee et al., 2012).
} 
Missbrauchsabbildungen angibt, mindestens einmal unmittelbar gegen einen jungen Menschen sexualisiert übergriffig geworden zu sein (Franke \& Graf, 2016). Die klinische Sexualforschung beschäftigt sich vor diesem Hintergrund mit der Frage, ob und unter welchen Umständen der Besitz und Konsum von Missbrauchsabbildung das Risiko erhöhen, hands-on übergriffig respektive rückfällig zu werden (ebd.). Der Ausschnitt aus dem familienpsychologischen Gutachten knüpft offensichtlich an diesen Diskurs an. Dabei wird zuerst auf ein Rückfallrisiko Arthur M.s eingegangen, da dieser bereits für sexuellen Kindesmissbrauch verurteilt wurde. Test- und Fragebogenuntersuchungen zu Kontrollüberzeugung - also Annahmen über die Steuerbarkeit von Ereignissen - Impulsivität und Opferempathie sind in einem solchen Zusammenhang übliche Instrumente (Henry et al., 2010). Die dort erreichten Ergebnisse führen den*die Gutachter*in in unserem Fall zu der Annahme, dass der Mann ein geringes Rückfallrisiko aufweise. Die festgestellten mittelbaren Formen von Dissexualität in digitalen Kontexten - vermutlich der Konsum von Missbrauchsabbildungen - werden nicht näher im Zusammenhang mit dem Rückfallrisiko erörtert. Es entsteht der Eindruck, als könne eindeutig zwischen On- und Offline-Kontexten unterschieden werden. Formen der Dissexualität seien dabei auf nur einen Kontext (nämlich online) beschränkt. Hierin liegt ein doppelter fachlicher Irrtum des Gutachtens. Erstens müssen Offline- und Online-Kontexte mitunter durch die Allgegenwart digitaler Medien zunehmend als Kontinuum und nicht zwei getrennte Realitäten betrachtet werden (Vobbe \& Kärgel, im Druck). Zweitens ist der Konsum von Missbrauchsabbildungen oder „Gewaltpornografie“ bei Menschen, die bereits sexualisiert gewalttätig wurden, eher ein Prädiktor für weitere Gewalttaten im Kontakt mit Kindern und Jugendlichen (Franke \& Graf, 2016). Diese Zusammenhänge übersieht das Gutachten. Es legt stattdessen die Mittelbarkeit der Dissexualität in einem scheinbaren Parallelraum als Verhaltensverbesserung aus. Ob dem die Annahme zugrunde liegt, Arthur M. baue durch den Konsum von Missbrauchsabbildungen Spannungen ab, kann nur spekuliert werden.

In dem Online-Offline-Kontinuum beachtet das Gutachten ebenso wenig die Implikationen des Besitzes ,,auffälligen“ Bildmaterials der 3-Jährigen durch einen Mann, der offenbar Missbrauchsabbildungen konsumiert und zur Risikogruppe für Hands-on-Gewalt gerechnet werden muss. Jenseits einer Strafwürdigkeit der Fotoaufnahmen ist streitbar, wie das Gutachten differenzieren will, dass Arthur M. zwar Missbrauchsabbildungen aus dem Internet konsumiert, nicht aber zu Aufnahmen der Tochter seiner Partnerin masturbiert. Franke und Graf (2016) konstatieren, dass es eine Herausforderung sei, mittels Diagnostik in gerichtlichen Verfahren zu vergleichbaren Einschätzungen zu kommen, wenn die Beurteilungsgrundlage Selbstauskünfte der Begutachteten sind. Schließlich belasten sich die wenigsten Menschen vor Gericht mehr als nötig. Da die Fotoaufnahmen Linas nicht im Verhältnis zur 
Dissexualität gesehen werden, scheint die Beurteilungsgrundlage des Gutachtens die Selbstauskunft Arthur M.s zu sein. Es ignoriert faktische Hinweise auf eine Viktimisierung des Mädchens. Kuhle et al. (2018) stellen außerdem fest, dass die Prävention des Konsums und der Verbreitung von Missbrauchsabbildungen eine Bandbreite bildlicher Darstellungen berücksichtigen müsse, die zur Selbstbefriedigung eingesetzt werden, teils aber (gezielt) unterhalb der Strafbarkeitsgrenze liegen.

Das Gutachten ignoriert weiterhin Implikationen, die sich daraus ergeben, dass sowohl die Kindesmutter als auch der Partner im Besitz der Aufnahmen sind. Im dargestellten Ausschnitt wird Arthur M. durch die Feststellung, dass er nicht alleiniger Besitzer der Aufnahmen sei, scheinbar entlastet. Tatsächlich belegt der Umstand jedoch, dass bereits eine Verbreitung begonnen hat. Entweder hat die Kindesmutter Arthur M. die Fotos gesendet oder umgekehrt. In beiden Fällen stellt sich die Frage nach dem Problembewusstsein, dem Motiv einer Urheberschaft sowie dem Motiv der Weiterleitung. Eine darüberhinausgehende Verbreitung kann überdies nicht ausgeschlossen werden. Gottfried et al. (2020) verweisen etwa darauf, dass Missbrauchsabbildungen von Konsument*innen geteilt werden, um im Tausch selbst an neue zu gelangen.

Nachfolgend werden Handlungsempfehlungen dargestellt, die sich aus der Kritik am Sachverständigengutachten ergeben.

\subsection{Handlungsempfehlungen (Teil 2)}

\subsubsection{Relativierung, Anfechtung oder Ablehnung des Gutachtens}

Das Gutachten ist fachlich zweifelhaft. Es integriert eine strafrechtliche Bewertung des Fotomaterials und orientiert sich offensichtlich am Grundsatz ,in dubio pro reo“, der nicht für familiengerichtliche Verfahren gilt. Damit überschreitet der*die Gutachter*in seine*ihre Kompetenzen. Besonders die Schlussfolgerungen zur mediatisierten Übergriffigkeit (Dissexualität) des Partners der Kindesmutter sind inhaltlich defizitär. Unterschiedliche Risikohinweise (mittelbare Dissexualität als Prädikator von Hands-on-Gewalt bei einem bereits gewalttätig gewordenen Mann; Besitz und Verbreitungsrisiko von augenscheinlich grenzverletzenden Darstellungen einer 3-Jährigen) werden unzureichend kombiniert. Dabei muss im familienpsychologischen Gutachten nicht der Zweifel an einer Gefahr ausgeschlossen, sondern zu erwartende positive oder negative Auswirkungen auf die Entwicklung der Kinder erörtert werden. Das Gutachten verfehlt diesen 
Anspruch. Dazu trägt maßgeblich bei, dass der*die Gutachter*in eine vermeintliche Dissexualität, also ein dem psychiatrischen Konzept der Dissozialität entlehntes Konstrukt, auf einen scheinbaren Parallelraum (digitale Kontexte) beschränkt. Mediatisierte sexualisierte Gewalt wird häufig dieserart bagatellisiert (Kärgel \& Vobbe, 2020).

Das Gutachten sollte im Rahmen des familiengerichtlichen Verfahrens deshalb entweder durch ergänzende Expertise oder Eindrücke weiterer (Fach-)Personen, zum Beispiel der Mitarbeiter*innen des Jugendamts oder spezialisierter Fachberater*innen, relativiert werden. Alternativ könnte es gänzlich angefochten werden, beispielsweise durch den Kindesvater oder eine rechtliche Vertretung der Kinder, etwa eine Verfahrenspflegschaft. Idealerweise wird das Gutachten direkt vom Gericht wegen fachlicher Mängel als unverwertbar abgelehnt. Zwar kommt Gutachten im Gerichtsprozess zumeist eine zentrale Bedeutung zu, die sich aus dem Beweisbeschluss herleitet. Nichtsdestotrotz sind sie nur eines von unterschiedlichen Mitteln der Beweisaufnahme (Bergmann, 2016). Sofern im konkreten Fall keine Unverwertbarkeit durch das Gericht festgestellt wird, sollten dringend ergänzende Perspektiven gehört werden. Es stellt keine fundierte Basis zur Entscheidung über den Schutz des Kindeswohls im Umfeld der Kindesmutter dar.

\subsubsection{Kindliche Beteiligung am Verfahren und Verfahrensbeistand}

Eine persönliche Anhörung von Kindern ist auch in familiengerichtlichen Sorgerechtsverfahren und Verfahren wegen Gefährdung des Kindeswohls geboten. Die Bundeskoordinierung Spezialisierter Fachberatung gegen sexualisierte Gewalt in Kindheit und Jugend (BKSF) (2020) stellt in einem Positionspapier zum Abschlussbericht der Kommission Kinderschutz Baden-Württemberg fest, dass diese Praxis sehr unterschiedlich gehandhabt wird. Ursächlich hierfür sei, dass die Anhörung von Kindern in die Gefährdung des Kindeswohls betreffenden Angelegenheiten in $\S 159$ Abs. 2 FamFG nicht ausdrücklich genug genannt werde. Voraussetzungen eines Gelingens ihrer Partizipation seien

- die altersentsprechende Befragung von Kindern durch eine qualifizierte Person,

- der Einsatz von Video-Ton-Aufzeichnungen, um belastende Mehrfachbefragungen zu vermeiden, 
- die obligatorische Bestellung und Beiordnung von Verfahrensbeiständen, welche den Kindeswillen und das Kindeswohl authentisch vor Gericht vertreten (ebd.).

In diesem Sinne kann auch Finn, gegebenenfalls Lina, am Verfahren teilhaben. Bestätigt Finn beispielsweise die Äußerungen, nackt vom Partner der Kindesmutter fotografiert worden zu sein, widerlegte dies die Einschätzung, dass sich ein sexualisiert übergriffiges Verhalten auf Online-Kontexte reduzieren ließe. Der Wille des Jungen würde darüber hinaus berücksichtigt werden können, insbesondere wenn er die Möglichkeit bekäme, sich so klar zu positionieren, wie vom Vater im ersten Fallausschnitt dargestellt. Grundsätzlich können Verfahrensbeistände auch Rechtsmittel für die von ihnen begleiteten Kinder einlegen, zum Beispiel Beschwerden gegen Entscheidungen des Gerichts, die auf Basis eines inadäquaten Gutachtens getroffen werden (Prenzlow, 2018).

\subsubsection{Beschränkungen des Umgangs zwecks Abklärung einer möglichen Gefährdung}

Ungeklärt ist weiterhin die Rolle der Kindesmutter im Zusammenhang eines mediatisierten sexuellen Kindesmissbrauchs Finns und Linas. In den Focus Group Interviews wird bereits die Anrufung des Familiengerichts als Hinweis auf einen Verstoß der Kindesmutter gegen Absprachen mit dem Jugendamt gewertet.

„In irgendeiner Form hat sie nicht kooperiert, deswegen gibt's diesen Antrag auf entsprechenden Eingriff.“

Rechtswissenschaftlerin, FGI

Die Entscheidung über daraus resultierende Konsequenzen für das Sorge- und Umgangsrecht der Mutter obliegt jetzt dem Familiengericht. Wird eine Gefährdung durch die Mutter festgestellt, können befristet begleitete Umgangskontakte nach $\S 1684$ Abs. 4 Satz 3 und 4 BGB ergänzend zu Beschränkungen des Aufenthaltsbestimmungsrechts angeordnet werden. Bei den begleiteten Umgangskontakten ist es dem Jugendamt möglich, vertiefend zu klären, wie die Beteiligung der Mutter an den Übergriffen einzuschätzen ist, gegebenenfalls beratend auf sie einzuwirken sowie zu prüfen, wie sich ihre Problemeinsicht und Erziehungsfähigkeit weiterentwickeln. Zwar würde der begleitete Umgang keinen niedrigschwelligen 
Eingriff in das Umgangsrecht der Mutter bedeuten. Letzterem steht jedoch die Beteiligung an oder Billigung von mediatisierter sexualisierter Gewalt gegenüber, sowie die Besorgnis, dass die Kindesmutter sich an einer Verbreitung grenzverletzender Fotos ihrer Kinder beteiligt. In derartigen Fällen darf die Maßnahme als gerechtfertigt erachtet werden (Güthoff, 2017). Anders gestaltet sich die Situation freilich, wenn für das Gericht ersichtlich wird, dass die Kindesmutter das für Finn und Lina bestehende Risiko versteht und Kontakte zum Partner unterbindet.

\subsubsection{Qualifikation von Familiengerichten}

Die Wirksamkeit von Handlungsempfehlungen gegen sexualisierte mediatisierte Gewalt ist begrenzt, solange sie sich auf jene Fachbereiche beschränkt, die im Kontext aneinander gekoppelter Systeme - hier Kinder- und Jugendhilfe an das Familienrecht - über das geringere Maß an Deutungs- und Entscheidungsmacht verfügen. Aus den Falldokumentationen des HUMAN-Projekts geht hervor, dass sich spezialisierte Fachberatungsstellen und Einrichtungen der öffentlichen Kinder- und Jugendhilfe nach anfänglich gelungener Krisenintervention angesichts schwer nachvollziehbarer gerichtlicher Entscheidungen machtlos fühlen.

„Das Jugendamt äußerte Bedenken bezüglich der gefundenen Missbrauchsabbildun-
gen. [...] Es sei ein rechtspsychologisches Gutachten in der Sache in Auftrag gegeben
worden. [...] Die Funde von der Tochter waren in einer ,Grauzone“ und die Zuordnung
zu Kinderpornografie konnte nicht eindeutig festgestellt werden.“

Falldokumentation 10.1

Das Problem wird durch die Mediatisierung sexualisierter Gewalt begünstigt, da diese als zumeist komplexes Phänomen zu Verwirrung und Handlungsunsicherheit $\mathrm{zu}$ führen scheint. Inwieweit zweifelhafte Entscheidungen mit den oft beklagten methodischen Mängeln der Begutachtung selbst zusammenhängen (Salewski et al., 2012) oder mit der interdisziplinären Problematik von „,Beweisbeschluss“ und „Anknüpfungstatsache“ (Kannegießer et al., 2020), kann von uns empirisch nicht nachvollzogen werden. Als dringend erforderlich erachten wir aber verbindliche Standards bezüglich der spezifischen Qualifikation von Sachverständigen und Familienrichter*innen. Das Gesetzespaket zur „Bekämpfung sexualisierter Gewalt gegen Kinder" greift diesen Ansatz zumindest teilweise auf. Mit den Ergänzungen bei $\S 23 b$ Abs. 3 GVG-E wird der Versuch unternommen, 
die Basiskompetenzen von Familienrichter*innen in relevanten Bezugsdisziplinen, insbesondere der Psychologie, zu verbessern. Keine Stellung bezieht der Entwurf jedoch zur Qualifikation von Sachverständigen. Dies ist insofern bemerkenswert, als eine regelmäßige Fortbildung von anderen beteiligten Gruppen, z. B. Verfahrensbeiständen, ausdrücklich gefordert wird (Bundesministerium für Justiz und Verbraucherschutz, 2021).

\section{Literatur}

Bange, D. (2011). Eltern von sexuell missbrauchten Kindern: Reaktionen, psychosoziale Folgen und Möglichkeiten der Hilfe. Hogrefe.

Bathke, S. A., Bücken, M., \& Fiegenbaum, D. (2019). Die Grundlagen: Kinderschutz, Kindeswohl und Kindeswohlgefährdung aus rechtlicher und fachlicher Perspektive. In S. A. Bathke, M. Bücken, \& D. Fiegenbaum (Hrsg.), Praxisbuch Kinderschutz interdisziplinär: Wie die Kooperation von Schule und Jugendhilfe gelingen kann (S. 5-106). Springer VS.

Bergmann, M. (2016). Der Beweisbeschluss im Kindschaftsverfahren: Schnittstelle zwischen Recht und Spekulation. FamRB, 14(9), 364-372.

Bley, R., Faber, M., Janke, L., Koeppen, J., \& Beckmann, P. (2020). Sexualdelikte im Wandel der Zeit. Fachhochschule für öffentliche Verwaltung, Polizei und Rechtspflege.

Bundeskoordinierung Spezialisierter Fachberatung gegen sexualisierte Gewalt in Kindheit und Jugend. 2020. Stellungnahme der BSKF zum Abschlussbericht der Kommission Kinderschutz in Baden-Württemberg. https://www.bundeskoordinierung.de/de/article/ 271.stellungnahme-der-bskf-zum-abschlussbericht-der-kommission-kinderschutz-inbaden-w\%C3\%BCrttemberg.html. Zugegriffen: 29. April 2021.

Bundesministerium für Justiz und Verbraucherschutz. (2019). Psychosoziale Prozessbegleitung: Wir begleiten Sie. https://www.bmjv.de/SharedDocs/Publikationen/DE/Psychosoz iale_Prozessbegleitung.pdf?_blob=publicationFile\&v=7. Zugegriffen: 26. Apr. 2021.

Bundesministerium für Justiz und Verbraucherschutz. (2021). Gesetzespaket zur Bekämpfung sexualisierter Gewalt gegen Kinder beschlossen. https://www.bmjv.de/SharedDocs/ Artikel/DE/2021/032521_GE_sexualisierte_Gewalt.html. Zugegriffen: 29. Apr. 2021.

Carter, D. L., Prentky, R. A., Knight, R. A., Vanderveer, P. L., \& Boucher, R. J. (1987). Use of pornography in the criminal and developmental histories of sexual offenders. Journal of Interpersonal Violence, 2(2), 196-211. https://doi.org/10.1177/088626087002002005.

Franke, I., \& Graf, M. (2016). Kinderpornografie: Übersicht und aktuelle Entwicklungen. Forensische Psychiatrie, Psychologie, Kriminologie, 10, 87-97.

Gerber, C. (2006). Was ist bei einer Kindeswohlgefährdung in Abgrenzung zum ASD der Aufgabenbereich der Polizei? In H. Kindler, S. Lillig, H. Blüml, T. Meysen, \& A. Werner (Hrsg.), Handbuch Kindeswohlgefährdung nach § 1666 BGB und Allgemeiner Sozialer Dienst (ASD). Eigenverlag.

Gottfried, E. D., Shier, E. K., \& Mulay, A. L. (2020). Child pornography and online sexual solicitation. Current Psychiatry Reports, 22(3), 10. https://doi.org/10.1007/s11920-0201132-y. 
Güthoff, F. (2017). Beschweren erlaubt. Beteiligungsmöglichkeiten für Kinder als fachliche Anforderung an den Kinderschutz. https://docplayer.org/33942637-Forum-1-friedhelmguethoff.html. Zugegriffen: 8. Juni 2021.

Henry, O., Mandeville-Norden, R., Hayes, E., \& Egan, V. (2010). Do internet-based sexual offenders reduce to normal, inadequate and deviant groups? Journal of Sexual Aggression, 16(1), 33-46. https://doi.org/10.1080/13552600903454132.

Hipp, T. N., Bellis, A. L., Goodnight, B. L., Brennan, C. L., Swartout, K. M., \& Cook, S. L. (2017). Justifying sexual assault: Anonymous perpetrators speak out online. Psychology of Violence, 7(1), 82-90. https://doi.org/10.1037/a0039998.

Igney, C., \& Monz, L. (2020). Wir vor Ort gegen sexuelle Gewalt. Trauma \& Gewalt, 14(3), 225-236. https://doi.org/10.21706/tg-14-3-225.

Kannegießer, A., Wegmann, U., \& Ebner, E. (2020). Abschlussbericht zum Pilotprojekt Professionelle Selbstkontrolle Online-Peer-Review-Verfahren. https://kompetenz-rpm.de/ gutachten/Abschlussbericht\%20_zum_Pilotprojekt_Kurzfassung_fh.pdf. Zugegriffen: 17. März 2021.

Kärgel, K., \& Vobbe, F. (2020). Mediatisierte Gewalt - Diffusion - Transzendenz: Erscheinungsformen und Herausforderungen sexualisierter Gewalt mit digitalem Medieneinsatz. Kindesmisshandlung und -vernachlässigung, 23(1), 30-43.

Kliemann, A., \& Fegert, J. (2015). Leitlinie der AG II des Runden Tisches zur Einschaltung der Strafverfolgungsbehörden. In J. Fegert \& M. Wolff (Hrsg.), Kompendium „Sexueller Missbrauch in Institutionen “. Entstehungsbedingungen, Prävention und Intervention (S. 486-500). Beltz Juventa.

Kuhle, L. F., Oezdemir, U., \& Beier, K. M. (2018). Sexueller Kindesmissbrauch und die Nutzung von Missbrauchsabbildungen. In K. M. Beier (Hrsg.), Pädophilie, Hebephilie und sexueller Kindesmissbrauch (S. 15-25). Springer VS.

Lee, A. F., Nien-Chen, L., Raina, L., Schuler, A., \& Prentky, R. A. (2012). Predicting handson child sexual offenses among possessors of internet child pornography. Psychology, Public Policy, and Law, 18(4), 644-672. https://doi.org/10.1037/a0027517.

Lillig, S. (2006). Wie ist mit der Neu-Meldung einer Kindeswohlgefährdung umzugehen? In H. Kindler, S. Lillig, H. Blüml, T. Meysen, \& A. Werner (Hrsg.), Handbuch Kindeswohlgefährdung nach § 1666 BGB und Allgemeiner Sozialer Dienst (ASD), Kapitel 47. Deutsches Jugendinstitut e. V.

Prenzlow, R. (2018). Bedeutung der Verfahrensbeistände in familiengerichtlichen Verfahren. Interessenvertretung der Kinder durch Verfahrensbeistände und Mitarbeiter des Jugendamtes. unsere jugend, 70(11+12), 458-465. doi: https://doi.org/10.2378/uj2018.art70d.

Riegel, D. L. (2004). Letter to the editor: Effects on boy-attracted pedosexual males of viewing boy erotica. Archives of Sexual Behavior, 33(4), 321-323. https://doi.org/10.1023/B: ASEB.0000029071.89455.53.

Salewski, C., Stürmer, S., Lotz-Schmitt, K., Kalhorn, E., Plato, J., Meyer, J., \& Rode, A.-K. (2012). Qualitätsmerkmale in der familienpsychologischen Begutachtung Untersuchungsbericht I. https://www.fernuni-hagen.de/psychologie/qpfg/pdf/Untersuchung sbericht1_FRPGutachten_1.pdf.

Schone, R. (2017). „Druck machen...“ - Zum neuen Miteinander von Jugendämtern und Familiengerichten bei der Abwendung der Kindeswohlgefährdung. Kindesmisshandlung und -vernachlässigung, 20(1), 12-31. 
Vobbe, F., \& Kärgel, K. (Im Druck). Hedonistic Utilitarianism: The strategic use of digital media along the online-offline continuum of sexualised violence. In L. Kuhle \& D. Stelzmann (Hrsg.), Sexual online grooming of children: Challenges for science and practice. Nomos (Erscheint voraussichtlich im Oktober 2021).

Open Access Dieses Kapitel wird unter der Creative Commons Namensnennung 4.0 International Lizenz (http://creativecommons.org/licenses/by/4.0/deed.de) veröffentlicht, welche die Nutzung, Vervielfältigung, Bearbeitung, Verbreitung und Wiedergabe in jeglichem Medium und Format erlaubt, sofern Sie den/die ursprünglichen Autor(en) und die Quelle ordnungsgemäß nennen, einen Link zur Creative Commons Lizenz beifügen und angeben, ob Änderungen vorgenommen wurden.

Die in diesem Kapitel enthaltenen Bilder und sonstiges Drittmaterial unterliegen ebenfalls der genannten Creative Commons Lizenz, sofern sich aus der Abbildungslegende nichts anderes ergibt. Sofern das betreffende Material nicht unter der genannten Creative Commons Lizenz steht und die betreffende Handlung nicht nach gesetzlichen Vorschriften erlaubt ist, ist für die oben aufgeführten Weiterverwendungen des Materials die Einwilligung des jeweiligen Rechteinhabers einzuholen.

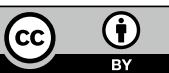

